\title{
Comparative analysis of hemodynamic parameters and heart rate variability in football and hockey players at the stage of sports improvement
}

\author{
Vladislav Lavrichenko ${ }^{1}$, Tatiana Lindt ${ }^{2}$, Irina Kalinina ${ }^{1 *}$, and Alexander Zolotarev ${ }^{1}$ \\ ${ }^{1}$ Kuban State University of Physical Culture, Sports and Tourism, 35015 Krasnodar, Russia \\ ${ }^{2}$ Siberian State University of Physical Culture and Sports, 644009, Omsk, Russia
}

\begin{abstract}
The article presents a multi-year multicenter research material concerning the study of adaptive aspects of hemodynamic and vegetative homeostasis of athletes engaged in football and hockey at the stage of sports improvement. The following was revealed: both groups of athletes were characterized by the formation of a cardiovascular functional system with a state of minimal tension of regulatory mechanisms to maintain hemodynamic homeostasis. The total sum of ICVR indicators in the group of football players was -1 point, which, according to the classification, can be assessed as a state of some functional tension, manifested by the mobilization of protective mechanisms. The hockey players were observed to have an optimal state of the regulation systems tension. It is characteristic of a satisfactory adaptation of the body to environmental conditions (1 point). The vascular type of self-regulation of blood circulation is more optimal in the formation of adaptation to dynamic loads of variable intensity.
\end{abstract}

\section{Introduction}

Hockey and football are the most difficult and emotional sports. As per sports physiology, motor activity of athletes engaged in these types is a dynamic work with variable intensity. During the game, there is a constant alternation of exercises that differ in duration, nature, and power in various ratios and sequences. The constant struggle for the puck and the ball is accompanied by a variety of complex movements. They for all other things, are carried out standing on skates for hockey players, and for football players are complicated by the influence of weather conditions.

The characteristic features of these games include: uneven physical activity during the game, which depends on the situation on the playground, the balance of forces of teams, the level of physical development and fitness of athletes [1-4]. The intensity of physical activity is determined by the intensity and duration of the game, the number of players on the court and ranges from maximum to moderate. The combination of short-term periods of relative rest with active actions of athletes on the playground is characteristic. The high intensity of playing the game is expressed in repeated changes in speed, rhythm, and direction of movement, which can be realized with a sufficient amount of energy generated by the body's functional systems using various components of metabolism [5-7].

In this case, the body experiences special stress when creating new functional systems. It can lead to various states of dysregulation. Especially often, such changes, up to sudden cardiac arrest are observed in sports such as football, hockey, marathon running, etc. Based on this, the processes of the football players and hockey players body adaptation during longterm training are of a similar nature. Pronounced changes occur on the part of the leading systems: cardiovascular, motor, and nervous. It determines the direction of our research. The hypothesis of the study was the assumption about the parallelization of the hemodynamic and vegetative homeostasis adaptation orientation in athletes engaged in game sports, in particular football and hockey.

\section{Materials and Methods}

The research was conducted on the basis of the Siberian state University of physical culture and sports research Institute of extreme conditions and the Department of theory and methodology of football and rugby of the Kuban state University of physical culture, sports and tourism. The study participants were 104 football players, 37 hockey players aged 17-18 years and 40 teenagers who are not engaged in physical culture and sports of the same age (control group). The study of the main parameters of hemodynamic and vegetative homeostasis was carried out using the REO-Spektr-2 hardware and instrument complex based on the Poly-Spektr program with preliminary measurement of body length and weight, with data input into a computer. Systolic and diastolic blood pressure in $\mathrm{mmHg}$ (APs and APd, respectively), as well as heart rate $\left(\mathrm{HR}\right.$ min $\left.^{-1}\right)$ were

\footnotetext{
* Corresponding author: kalininirina@yandex.ru
} 
recorded using an Omron MX tonometer. Other hemodynamic parameters were calculated using generally applicable methods. The index of cardiovascular regulation (ICVR) was determined by the method of N. I. Arinchin [8] in the modification of V. N. Karlov et al. [9]: when determining the ICVR, more than 110 points were considered as vascular type, less than 90 points it is cardiac type, 90-110 - mixed type of self-regulation of blood circulation.

Cardiointervalography and spectral analysis of heart rate were performed in accordance with the recommendations of the international standard variability heart rate (VHR) (1996). The characteristic of the initial vegetative tone with the determination of the leading mechanisms of regulation was normalized according to the method of A.M. Vane (2000), where $\mathrm{LF}>\mathrm{VLF}<\mathrm{HF}$ - unstrained vegetative balance, $\mathrm{VLF}>\mathrm{LF}<\mathrm{HF}-$ strained vegetative balance, $\mathrm{VLF}>\mathrm{HF}<\mathrm{LF}-$ sympathicotonia (relative or absolute), $\mathrm{LF}<\mathrm{HF}>\mathrm{VLF}$ - relative vagotonia [10, 11].

For the integral analysis of the heart rate functional properties, we used the method of calculating IARS (an indicator of the adequacy of regulation processes). It allows us not only to combine the methods of variational pulsometry, but also to give an adequate assessment of the functional systems components intersystem interaction $(10,11)$.

All studies were conducted in the morning time before performing training and physical activities in compliance with the basic bioethical and hygienic requirements, taking into account contraindications and testing rules. Statistic data processing was performed using the statistical software package STATISTICA 6.0.

\section{Results and Discussion}

Analysis of the studied indicators revealed the following: adaptive manifestations to long-term physical exertion on the part of hemodynamic homeostasis in football players are the formation of mixed (table. 1) and hockey players-vascular types of blood circulation regulation (respectively).

Table 1. Main hemodynamic and vegetative parameters of the studied groups $(\mathrm{M} \pm \mathrm{m})$

\begin{tabular}{|l|l|l|l|}
\hline Indicators & football Players $(\mathrm{n}=104)$ & hockey Players $(\mathrm{n}=37)$ & control Group $(\mathrm{n}=40)$ \\
\hline Heart rate, $\mathrm{min}^{-1}$ & $67,9 \pm 0,6$ & $64,3 \pm 0,8^{*}$ & $62,5 \pm 0,10^{\circ}$ \\
\hline $\mathrm{APs}, \mathrm{mm} \mathrm{Hg}$ & $107,6 \pm 1,0$ & $120,8 \pm 0,8^{*}$ & $118,2 \pm 0,10$ \\
\hline $\mathrm{APd}, \mathrm{mm} \mathrm{Hg}$ & $69,3 \pm 0,7$ & $74,1 \pm 0,8^{*}$ & $71,3 \pm 0,10$ \\
\hline APm mm Hg. & $82,0 \pm 0,7$ & $89,6 \pm 0,7^{*}$ & $86,9 \pm 0,01^{\circ}$ \\
\hline PP, mm Hg & $38,3 \pm 0,6$ & $46,8 \pm 0,7^{*}$ & $46,9 \pm 0,14^{\circ}$ \\
\hline Robinson Index, u.e & $73,0 \pm 0,8$ & $77,7 \pm 1,1^{*}$ & $82,9 \pm 0,21^{\circ}$ \\
\hline Systolic volume of the Heart, ml & $61,5 \pm 0,7$ & $69,4 \pm 0,7^{*}$ & $54,3 \pm 0,10^{\circ}$ \\
\hline Cardiac Qutput, l & $4,2 \pm 0,5$ & $4,5 \pm 0,1$ & $3,35 \pm 0,01^{{ }^{\circ}}$ \\
\hline Peripheral vascular resistance, u.e & $2036,0 \pm 5,4$ & $1659,2 \pm 35,6^{*}$ & $2192,8 \pm 11,5^{\circ}$ \\
\hline ICVR, u.e. & $92,5 \pm 0,3$ & $115,2 \pm 1,2^{*}$ & $110,0 \pm 0,5^{\circ}$ \\
\hline TP, $\mathrm{ms}^{2}$ & $3475,2 \pm 79,0$ & $7161,1 \pm 12,3^{*}$ & $5030,0 \pm 90,9^{\circ}$ \\
\hline VLF, ms & $886,7 \pm 29,1$ & $2274,3 \pm 66,2^{*}$ & $1359,7 \pm 25,3^{\circ}$ \\
\hline LF, $\mathrm{ms}^{2}$ & $1196,3 \pm 24,1$ & $1971,5 \pm 37,2^{*}$ & $1635,4 \pm 32,9^{\circ}$ \\
\hline HF, $\mathrm{ms}^{2}$ & $1503,4 \pm 61,0$ & $2916,2 \pm 90,1^{*}$ & $2491,4 \pm 87,7^{\circ}$ \\
\hline
\end{tabular}

Note: * - reliability of differences at $\mathrm{P}<0.05$ between the performance of football players and hockey players, ${ }^{\circ}$ - reliability of differences at $\mathrm{P}<0.05$ between the performance of the players and the control group; $\bullet$ - reliability of differences at $\mathrm{P}<0.05$ between the performance of hockey players and a control group

Nowadays, it is believed that athletes with vascular type of regulation are characterized by a good adaptation to long-term exposure to disturbing factors. It is manifested in a wide choice of interaction indicators necessary for maintaining homeostasis. For athletes with a mixed type, a balance of functional systems is characteristic not only in quantitative, but also in qualitative terms. It ensures high physical performance and increases the reserve capabilities of the circulatory system. Football players are characterized by significantly lower values of blood pressure, heart stroke volume, MV and higher values of peripheral vascular resistance, with the formation of a mixed type of blood circulation regulation. In hockey players who have characteristic signs of hemodynamic adaptation to high loads-vascular type. The data obtained in this study confirm our previous studies on a group of road cyclists. They indicate the formation of a hypokinetic type of blood circulation in athletes with vascular type. It is favorable for ensuring adaptation to muscle loads [12].

Vegetative reactivity in the group of football players was characterized as LF < HF $>$ VLF-relative vagotonia, in hockey players as $\mathrm{VLF}>\mathrm{LF}<\mathrm{HF}$-strained vegetative balance. It should be noted that in the group of athletes engaged in hockey, all the indicators of spectral analysis in their power were significantly higher in relation to those of football players.

When studying the integral PARS indicator, we used 5 components: assessment of the total effect of regulation (HR, M), heart automatism function $(\sigma, \Delta \mathrm{X}, \mathrm{V})$, vegetative homeostasis $(\triangle \mathrm{X}, \mathrm{AMO})$, stability of regulation $(\mathrm{M}, \mathrm{VLF})$ and activity of subcortical centers (LF, HF, VLF). It was found that according to the indicators that assess the total effect of regulation in both groups of athletes - normocardia, the automatism function is regarded as moderate sinus arrhythmia, which is typical for athletes of the sports improvement group. On the part of vegetative homeostasis, the state of football players is regarded as a moderate predominance of parasympathetic regulation, while hockey players have a pronounced predominance of parasympathetic activity. The stability of the regulation of football players was characterized by transients with a predominance of parasympathetic activity, which is due to the economy of energy and metabolic processes. In hockey players, a state of central type dysregulation was observed with the excitation of the central heart rate control circuit, which 
indicates a state of some overstrain. The activity of subcortical centers (cardiovascular and vasomotor) in football players was in the zone of normal values, for hockey players it was characterized as pronounced. The total sum of ICVR indicators in the group of football players was -1 point, which, according to the classification, can be assessed as a state of some functional tension, manifested by the mobilization of protective mechanisms. The hockey players were observed to have an optimal state of the regulation systems tension. It is characteristic of the body satisfactory adaptation to environmental conditions ( 1 point).

\section{Conclusions}

Thus, at the stage of sports improvement, both groups of athletes were characterized by the formation of a cardiovascular functional system with a state of regulatory mechanisms minimal tension to maintain hemodynamic homeostasis. Based on the obtained data on the superiority of the functional state in the group of hockey players, the vascular type of self-regulation of blood circulation is more optimal in the formation of adaptation to dynamic loads of variable intensity.

\section{References}

1. B.A. Ateş, Physical education of students, 23(5), 223-228 (2019). DOI: https://doi.org/10.15561/20755279.2019.0502

2. O.O. Podrigalo, O.V. Borisova, L.V. Podrigalo, S.S. Iermakov, V.V. Romanenko, O.V. Podavalenko, O.A. Volodchenko, J.O. Volodchenko, Physical education of students, 23(6), 313-319 (2019). DOI: https://doi.org/10.15561/20755279.2019.0606

3. F.A. Iordanskaya, Bulletin of sports science, 3, 33-38 (2010)

4. E.B. Kramer, L. Serratosa, J. Drezner, J. Dvorak, Br J Sports Med, 50(2), SI-83 (2016)

5. S.G. Priori, C. Blomström-Lundqvist, A. Mazzanti, N. Blom, M. Borggrefe, J. Camm et al. Eur Heart J, 36(41), $2793-2867$. DOI: 10.1093/eurheartj/ehv316 (2015)

6. K.G. Harmon, I.M. Asif, D. Klossner, J.A. Circulation, 123(15), 1594-1600 (2011). DOI: 10.1161/circulationaha.110.004622

7. L.M. Makarov, Russian Bulletin of Perinatology and Pediatrics, 62(1), 40-46 (2017)

8. N.I. Arinchin, Abstracts of the report conference based on the results of scientific research. works of BGOIFK for 1969 (Minsk, 101-103, 1970)

9. V.N. Karlov, A.F. Ershov, T.I. Shustov, Patent for invention No. SU 1713551, 1992

10. V.M. Mikhailov, Quantitative assessment of the level of health in restorative medicine (Neurosoft, Sochi, 2005)

11. A.S. Kuznetsov, Z.M. Kuznetsova, Russian Journal of Physical Education and Sport, 14(4), 5-7 (2019). DOI: 10.14526/20704798-2019-14-4-5-7

12. I.N. Kalinina, I.A. Booth, Physical culture and health, 3(67), 128-132 (2018) 